View Article Online / Journal Homepage / Table of Contents for this issue DUNSTAN, JOWETT, AND GOULDING :

\title{
CLIII.-The Rusting of Iron.
}

By Wyndham Rowland Dunstan, F.R.S., Hooper Albert Dickinson

Jowett, D.Sc., and ERnest Goulding, D.Sc.

Although the rusting of iron was at first regarded as a simple process of oxidation (Gmelin, Handbook of Chemistry, vol. v, p. 185), the explanation at present generally accepted is chiefly based upon the experiments of Crace Calvert (Manchester Lit. Phil. Mem., 
$1871,5,104$ ) as interpreted by Crum Brown (Journal of the Iron and Steel Institute, 1888, 129-131). Iron is supposed to undergo the change into rust through the combined action of the oxygen and carbonic acid of the air in the presence of liquid water, the carbonate or bicarbonate of iron first formed being gradually converted by the further action of atmospheric oxygen into a ferric hydroxide or rust. The successive changes were summarised by Crum Brown in the following equations :

$$
\begin{gathered}
4\left(\mathrm{Fe}+\mathrm{H}_{2} \mathrm{O}+\mathrm{CO}_{2}\right)=4 \mathrm{FeCO}_{3}+4 \mathrm{H}_{2} . \\
4 \mathrm{FeCO}_{3}+6 \mathrm{H}_{2} \mathrm{O}+\mathrm{O}_{2}=2 \mathrm{Fe}_{2}(\mathrm{OH})_{6}+4 \mathrm{CO}_{2} .
\end{gathered}
$$

The well-known action of alkalis in preventing the rusting of iron was thus explained by their power of absorbing carbonic acid, without which rusting was supposed to be unable to occur.

The results of experiments conducted for the purpose of explaining the rapid corrosion of iron and lead pipes by soft water led one of us to the conclusion that the nature of the chemical change involved in the atmospheric oxidation of metals was not fully explained by the current theory and the present investigation was then undertaken.

A preliminary account of some of the results which the investigation has furnished was included in a lecture on "The Rusting of Iron" delivered by one of us to the Royal Artillery Institution at Woolwich in 1898 (Dunstan, Proceedings of the Royal Artillery Institution, 1899, 26, No. 5). A brief account of the conclusions reached has also appeared in the Proceedings of the Chemical Society (Dunstan, Proc., $1903,19,150)$.

It has been proved that the aërial oxidation of iron can take place in the absence of carbonic acid and that some other explanation must therefore be found for the inhibiting effect of alkalis. A series of experiments was carried out with this object and the results suggested that the process of rusting may involve the formation of hydrogen peroxide. A careful investigation was therefore made, with the result that the conclusion is inevitable that although hydrogen peroxide cannot be actually detected during the rusting of iron, this compound is probably formed as an intermediate product of the change. The chemical reactions concerned in the process may be represented in outline by the following equations :

$$
\begin{gathered}
\mathrm{Fe}+\mathrm{O}_{2}+\mathrm{H}_{2} \mathrm{O}=\mathrm{FeO}+\mathrm{H}_{2} \mathrm{O}_{2} . \\
2 \mathrm{FeO}+\mathrm{H}_{2} \mathrm{O}_{2}=\mathrm{Fe}_{2} \mathrm{O}_{2}(\mathrm{OH})_{2},\left(\mathrm{Fe}_{2} \mathrm{O}_{3}, \mathrm{H}_{2} \mathrm{O}\right) .
\end{gathered}
$$

The excess of hydrogen peroxide immediately reacts with the iron forming a further quantity of rust.

$$
\begin{gathered}
\mathrm{Fe}+\mathrm{H}_{2} \mathrm{O}_{2}=\mathrm{FeO}+\mathrm{H}_{2} \mathrm{O} . \\
2 \mathrm{FeO}+\mathrm{H}_{2} \mathrm{O}_{2}=\mathrm{Fe}_{2} \mathrm{O}_{2}(\mathrm{OH})_{2},\left(\mathrm{Fe}_{2} \mathrm{O}_{3}, \mathrm{H}_{2} \mathrm{O}\right) .
\end{gathered}
$$


In support of these conclusions, it has been proved as the result of a series of experiments that many soluble substances, which decompose and therefore interfere with the existence of hydrogen peroxide, likewise prevent the rusting of iron. The analysis of a number of specimens of iron rust has shown that its composition may be fairly represented by the formula $\mathrm{Fe}_{2} \mathrm{O}_{2}(\mathrm{OH})_{2}$. The conclusion that hydrogen peroxide is formed in the process of rusting receives strong support from the evidence accumulated by other observers that this compound is frequently produced in those chemical changes which involve spontaneous oxidation through the agency of the oxygen of the air.

The formation of hydrogen peroxide thus appearing to be a necessary part of the chemical process of rusting, the nature of this process required investigation. The formation of hydrogen peroxide during various processes of oxidation has been explained, notably by HoppeSeyler in connection with physiological processes, by the supposition that the substance is oxidised by one atom of a molecule of oxygen, the other atom of which attaches itself to a molecule of water forming hydrogen peroxide. Thus in the case of iron the initial change would be

$$
\mathrm{Fe}+\mathrm{O}_{2}+\mathrm{H}_{2} \mathrm{O}=\mathrm{FeO}+\mathrm{H}_{2} \mathrm{O}_{2} \text {. }
$$

On the other hand, Traube has supposed that the oxygen is taken, not from molecular oxygen, but from one molecule of water, the liberated hydrogen combining with a molecule of oxygen to form hydrogen peroxide. Thus, in the case of iron, $\mathrm{Fe}+\mathrm{OH}_{2}+\mathrm{O}_{2}=\mathrm{FeO}+\mathrm{H}_{2} \mathrm{O}_{2}$, Traube's view involves the assumption that hydrogen peroxide is not oxidised water, but " reduced" or hydrogenised oxygen, and in support of this contention he has brought forward a considerable body of evidence.

The two possible modes of formation of hydrogen peroxide have been experimentally investigated so far as they relate to the rusting of iron. Positive evidence has been obtained in support of the theory involving the decomposition of water, whilst negative evidence was forthcoming against the view that oxygen is taken directly from dissolved oxygen. The results distinctly support the conclusion that water is decomposed by the iron and that the liberated hydrogen goes to form hydrogen peroxide with the dissolved molecular oxygen. If the existence of hydrogen peroxide is prevented by the introduction of a soluble substance capable of destroying it, little or no action between the iron and the water takes place at the ordinary temperature. The oxidation process appears therefore to be a part of a definite cycle of chemical change, the energy of which is partly derived from the combination of the hydrogen formed. It bas been found that rusting of 
iron can occur in the absence of free oxygen provided that certain oxidising agents are present with which the hydrogen of the water can interact.

Another possible explanation of the formation of hydrogen peroxide in the rusting process may be noticed here. It has often been suggested that hydrogen peroxide may be formed from oxygen dissolved in water, especially under the influence of light. If this were proved to be the case, the formation of hydrogen peroxide by the direct oxidation of water would be established and an extremely simple explanation afforded of the phenomena of the oxidation of iron. There is, however, no satisfactory recorded evidence that hydrogen peroxide is ever produced in a solution of oxygen in water, whilst rusting commences and proceeds without interruption in the dark.

An attempt has been made by Whitney (J.Amer. Chem. Soc., 1903, $25,394)$ to apply the theory of electrolytic dissociation to the explanation of the rusting of iron. Water being assumed to be slightly dissociated into hydrogen and hydroxyl ions, it should be capable of dissolving minute quantities of metallic iron, owing to the formation of an electric circuit containing iron as the positive and hydrogen as the negative element. If this is the case, the rusting process would be explained, the ferrous hydroxide first formed absorbing oxygen from the air. Substances such as alkaline salts interfere with, or altogether prevent rusting by hindering the accumulation of hydrogen ions, whilst acids and certain salts tend to accelerate rusting by increasing the accumulation of hydrogen ions. When the concentration of the hydrogen has attained a certain maximum, the hydrogen, according to Whitney, is evolved as a gas. This is, however, contrary to fact, no hydrogen being liberated in ordinary circumstances during the rusting of iron. Careful experiment has also failed to confirm Whitney's statement that iron dissolves to a slight extent in water, whilst the theory in question is shown to afford no explanation of the fact, established during the course of this investigation, that substances other than alkaline salts, such as chromic acid and potassium dichromate, prevent the rusting of iron.

In order to gain accurate information concerning the nature of the chemical process of rusting, experiments were made in the first instance with highly purified materials. The presence of impurities in the iron has a considerable influence in modifying the action and in many cases of accelerating the rusting. This subject has been discussed in connection with steel in the report on Corrosion made by one of us to the Steel Rails Committee of the Board of Trade (1900).

The part played by carbonic acid during atmospheric oxidation of iron must, in future, be regarded as subsidiary and not essential. The presence of carbonic acid accelerates the rusting process and 
forms a carbonate of the metal to an extent depending on the amount of carbonic acid present. Since this carbonate is gradually decomposed by the further action of water and oxygen, only a minute proportion is found in rust which has been long exposed to air. As the carbonic acid at first absorbed in the action is afterwards evolved again by the decomposition of the carbonate, it is obvious that a small proportion may produce a considerable effect in accelerating the action. It is evident, however, that the main action concerned in the ordinary atmospheric oxidation of iron is not dependent on the presence of carbonic acid, but is a change proceeding between oxygen and iron in the presence of liquid water.

The investigation has been partially extended to other metals, with the general conclusion that the atmospheric oxidation of all the metals would appear to be conditioned by the same process as that which has been traced in the case of iron. In many instances, as in those of lead and zinc, the formation of hydrogen peroxide can be actually detected during the course of the action, and it is found that the action in these cases is stopped by those agents which also prevent the atmospheric oxidation of iron.

In the ordinary rusting of impure iron, three factors may be involved, namely, (1) the direct oxidation process, which depends on the action on the iron of oxygen together with liquid water, (2) the action of atmospheric carbonic acid, and (3) electrolytic action generated by impurity. Each of these factors has been studied separately and is discussed in the following pages.

\section{Action of Dry and Moist Gases on Iron.}

The first experiments were carried out with the object of ascertaining the behaviour of metallic iron (i) in the presence of dry gases and (ii) in the presence of moist gases. Small pieces of highly purified iron (shown by analysis to contain 99.94 per cent. of iron) were placed in contact with the following gases: oxygen, carbon dioxide, a mixture of oxygen and carbon dioxide, a mixture of oxygen and ammonia. In one series of experiments, the gases were carefully dried, whilst in the other series they were saturated with water vapour. The experiments were conducted in the following manner. The plate of iron, after being polished, was placed in a piece of hard glass tubing, contracted at either end, which was then heated to redness and a current of pure hydrogen passed over it. While the iron was still hot, one end of the tube was sealed and the metal was allowed to cool in the atmosphere of hydrogen. The tube $A$ was now attached to the apparatus shown in Figure 1, and was exhausted by means of a Sprengel pump, the tap $d$ being closed. The particular gas required 
was then admitted by the $\operatorname{tap} d$. The tube was again exhausted and again filled with the gas, this operation being repeated several times in order to remove the whole of the hydrogen. Finally the tube filled with the gas was sealed at $f$. The gases employed were carefully purified and in series $\mathrm{i}$ were finally dried by being passed through a long tube containing phosphoric oxide, whilst in series ii they were saturated with aqueous vapour by being bubbled through air-free water. In order to ensure that no water should be deposited on the surface of the metal by condensation due to fluctuation of temperature, the tubes were placed in a thermostat, the temperature of which was maintained at $34^{\circ}$, and were left for three months. At the end of this time the tubes were examined, and not the slightest trace of rust was found in any case.

A precisely similar set of tubes was prepared and allowed to remain

FIG. 1.

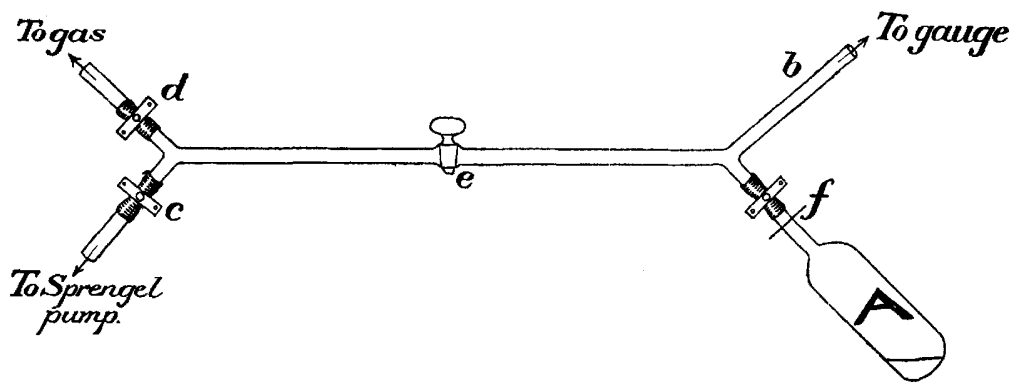

at the ordinary variable temperature. The results of these two series of experiments may be summarised thus :

At constant temperature. At variable temperature.

Dry gases........................ No visible action. No visible action.

Moist oxygen ......................... No visible action. Large amount of rust formed.

Moist oxygen and carbon dioxide No visible action. Copious rusting.

Moist oxygen and ammonia ...... No visible action. No visible action.

Moist carbon dioxide ............... No visible action, No visible action.

It is therefore proved that rusting of iron does not occur in the presence of either dry or moist oxygen, carbon dioxide, or in a mixture of these gases if the temperature is constant. When, however, the temperature fluctuates and liquid water is deposited on the metal, rusting occurs in the presence of oxygen alone or mixed with carbon dioxide, but not in carbon dioxide alone or in oxygen mixed with ammonia.

Since these results, especially with reference to the action of carbon dioxide, are at variance with those of Crace Calvert (loc. cit.), which are now generally accepted, the experiments described by that chemist 
were repeated under the same conditions. Carefully cleaned pieces of iron, having a gutta-percha mass at one end, were introduced into tubes which were placed over a trough of mercury, and the air in the tubes was displaced by leading in the desired gases. The following results were obtained :

\begin{tabular}{|c|c|c|}
\hline Experiment. & Result. & Crace Calvert's result. \\
\hline $\begin{array}{l}\text { Pure oxygen and aqueous } \\
\text { vapour. }\end{array}$ & $\begin{array}{l}\text { After some weeks rusted } \\
\text { locally, especially at the } \\
\text { edges. }\end{array}$ & $\begin{array}{l}\text { "In three experiments } \\
\text { only one blade slightly } \\
\text { oxidised." }\end{array}$ \\
\hline $\begin{array}{l}\text { Oxygen, carbon dioxide, } \\
\text { and aqueous vapour. }\end{array}$ & $\begin{array}{l}\text { After a few weeks specks } \\
\text { of rust appeared. }\end{array}$ & $\begin{array}{l}\text { "Oxidation most rapid, a } \\
\text { few hours being suf- } \\
\text { ficient. The blade as- } \\
\text { sumed a dark green } \\
\text { colour which then } \\
\text { turned brown-ochre." }\end{array}$ \\
\hline $\begin{array}{l}\text { Carbon dioxide and aque- } \\
\text { ous vapour. }\end{array}$ & Iron remained quite bright. & $\begin{array}{l}\text { "Slight appearance of a } \\
\text { white precipitate found } \\
\text { to be carbonate of iron. } \\
\text { Two only out of six ex- } \\
\text { periments did not give } \\
\text { these results." }\end{array}$ \\
\hline
\end{tabular}

These results are seen to differ from those of Crace Calvert whilst they confirm those we had already obtained, the slight variations being due to the higher laboratory temperature in the later series.

It is not possible from the description given by Crace Calvert of his experiments to state the origin of the difference between his results and ours. It is clear, however, that insufficient precautions were taken by him to avoid more than one important source of error, in particular to control the effect of a fluctuating temperature.

Our experiments therefore lead to the following conclusions. Iron does not rust when left in contast with dry gases. In the case of moist gases, no rusting occurs with oxygen or with mixtures of oxygen and carbon dioxide so long as a constant temperature is maintained, but if the temperature is allowed to fluctuate, liquid water condenses on the surface of the iron and rust is produced. The presence of liquid water is shown to be necessary for rusting to take place, a point of importance to which attention was called by Crum Brown (loc. cit.).

The subsidiary action of carbon dioxide in connection with Crace Calvert's experiments may be conveniently discussed later in this paper.

\section{The Action of Oxygen and Liquid Water on Iron.}

In order to investigate the action of pure oxygen on iron in presence of water, experiments were carried out in which the oxygen and water were both carefully purified in such a manner as to ensure their freedom from carbon dioxide. The iron, the surface of which had been thoroughly cleaned and polished and any trace of oxide 
afterwards reduced in a current of hydrogen, was contained, as in previous experiments (page 1553), in the tube $A$, which was attached at $c$ by the only india-rubber joint in the whole apparatus (Figure 2). Hydrogen, carefully freed from carbon dioxide by passing it through several wash-bottles and towers contrining potassium hydroxide, was driven from $B$ through the distilling flask $C$, which contained water, lime, and potassium permanganate. The other parts of the apparatus corresponded with those used in the previous experiments. The whole apparatus was first filled with hydrogen gas, the clamp $c$ being closed. A quantity of water amounting to about 100 c.c. was then distilled in a current of hydrogen from the flask $C$ into the bottle $D$. The whole apparatus being still full of hydrogen, the taps $b$ and $d$ were closed, $c$ was opened, and the portion of the apparatus above tap $b$ was exhausted by means of the air-pump. The taps $c$ and $a$ were now

FIG. 2.

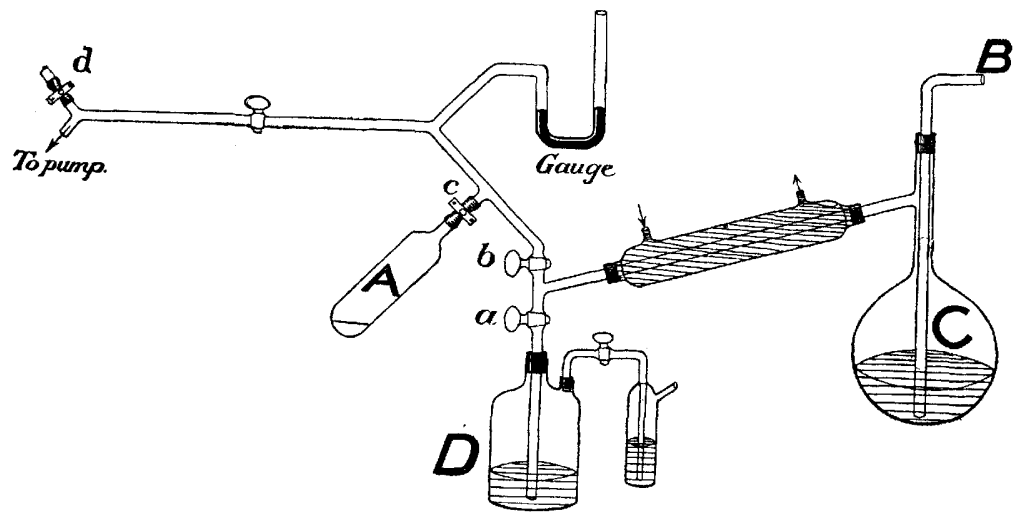

closed, tap $b$ was opened, and water was distilled into the T-piece above tap $a$ until the level of the distilled water reached tap $c$. On opening tap $c$, the water saturated with pure hydrogen entered the tube $A$ and completely covered the piece of iron. The $\operatorname{tap} b$ was now closed and the portion of the apparatus above this tap was filled with pure oxygen, admitted through tap $d$. The utmost care was taken that this oxygen was completely free from carbon dioxide by passing it through three wash-bottles containing aqueous potassium hydroxide and afterwards through a tower of solid potassium hydroxide. The apparatus above $b$ was re-exhausted and again filled with the gas, as in the experiments previously described.

When the iron was left in contact with water saturated with hydrogen and in an atmosphere of the same gas, no action took place. On admission of oxygen, however, action immediately commenced, a 
substance of a green colour being produced which rapidly changed to the red colour characteristic of rust.* This experiment was repeated several times, always with the same result. In other experiments, nitrogen was used instead of hydrogen, similar results being obtained. The action was the same whether light was excluded or not.

The "rust" produced in these experiments was analysed by the following method, which enables the iron, hydrogen, and oxygen to be determined in a very small quantity of the substance. A weighed quantity of the air-dried rust was heated in a porcelain boat in a current of dry carbon dioxide and the loss due to combined water thus determined. The residue was reduced by heating it in a stream of hydrogen, and the resulting water was collected and weighed and the amount of oxygen calculated from the result. The total iron in the residue was estimated gravimetrically in the usual way. Analyses of two different specimens furnished the following results :

\begin{tabular}{lrr} 
& \multicolumn{1}{c}{ I. } & \multicolumn{1}{c}{ II. } \\
Iron $\ldots \ldots \ldots \ldots \ldots$ & $62 \cdot 16$ & $62 \cdot 2$ \\
Hydrogen ........... & $1 \cdot 14$ & $1 \cdot 2$ \\
Oxygen ............ & $35 \cdot 97$ & $36 \cdot 6$
\end{tabular}

\section{Action of Water Alone on Iron.}

It has been assumed by Whitney (loc. cit.) that water, on the electrolytic hypothesis, being slightly dissociated, is capable of dissolving iron in the absence of oxygen or of carbon dioxide owing to the formation of a voltaic couple in which the iron acts as the positive element, whilst the negative element consists of the few hydrogen ions which the water normally contains. On this supposition he has founded a theory of rusting. He shows that theoretically those substances, such as acids, which permit of the concentration of hydrogen ions accelerate rusting, whilst those substances, such as alkalis, which diminish the concentration of the hydrogen ions inhibit rusting. The experimental evidence adduced by Whitney in support of the fundamental assumption that iron dissolves appreciably in pure water in the absence of oxygen or carbonic acid is slender and unsatisfactory. The theory also involves the assumption that hydrogen is liberated during the rusting of iron, but no evidence of the formation of free hydrogen is produced by Whitney, and we have never noticed its production in any of the experiments we have conducted. As regards the solubility of iron in water, Whitney describes an experiment in which a piece of

* The course followed in the oxidation of the surface of the metal is subject to considerable variation, the action being often localised in patehes. As these variations are noticed with nearly pure iron, they are probably connected with the physical condition of the surface and not with impurity. 
bright iron was placed in a bottle of boiling water, and, while the water was still boiling, a stopper carrying a glass tube was firmly inserted in the neck of the bottle and the end of the glass tube sealed in the blowpipe; the stopper and the neck of the bottle were afterwards coated with paraffin wax. In experiments thus made, he states that the iron remained without change for weeks, but, on admitting air, rust was formed in a few minutes, the water becoming cloudy and assuming a yellow colour. In 15 or 20 minutes, rust was produced throughout the bottle, being deposited on the glass as well as on the metal. From these results, Whitney concludes that the iron had dissolved in the water before the admission of air, and that the oxygen admitted reacted with the dissolved iron with the formation of rust.

We have repeated this experiment in the following manner. A flask of 600 c.c. capacity, fllled with distilled water, was boiled for 15 minutes; two pieces of purified iron each about $1 \frac{1}{2}$ inches square were then placed in the flask, and an india-rubber stopper carrying a glass tube which projected $7-8$ inches above the stopper and ended in a capillary was fitted into the neck of the flask, the water being kept boiling continuously. The water was allowed to boil for five minutes longer, when the capillary was sealed and the stopper coated with paraffin wax. This flask was left at the ordinary temperature for three weeks, in the course of which no visible change occurred. It was then opened, when one-half of the liquid was quickly poured into a beaker, the other half being left in contact with the iron in the flask. The liquid in the beaker on exposure to the air showed no cloudiness, no yellow coloration, and no separation of rust. In fact, on testing the liquid for iron by the extremely delicate thiocyanate reaction not a trace could be detected. The pieces of iron in the open flask after an hour showed signs of rusting, just as in ordinary cases, but the phenomena described by Whitney were not observed. We are tinerefore unable to confirm Whitney's statement that liquid water alone is capable of dissolving even an infinitesimal quantity of iron. This being the case, the theory based on this statement becomes untenable.

It having been proved that iron rusts in the presence of oxygen and water without the aid of carbon dioxide, it follows that the inhibitive action of alkalis on the process of rusting must find some other explanation than that hitherto accepted, which assumes that alkalis remove carbon dioxide, in the absence of which rusting cannot occur. The action of a number of alkalis and salts on rusting has therefore been carefully studied. 


\section{Effect of Salts and Other Substances on the Process of $R u s t i n g$.}

The influence of various salts on the oxidation of iron was studied by placing pieces of highly purified polished iron in a solution of the salt standing in an atmosphere of oxygen. The experiments were carried out by the methods already described, with the following modification (Figure 3). The tube $A$ containing the salt and metal is connected with the condenser at $a$ and also with a guard-tube containing a solution of potassium hydroxide. The water is distilled into $A$ in a current of hydrogen, and one arm of the tube $A$ is then sealed at $b$; it is then attached by the other arm to the apparatus previously described, is exhausted several times, and filled with the gas required. The tube is then sealed off.

FIG. 3.

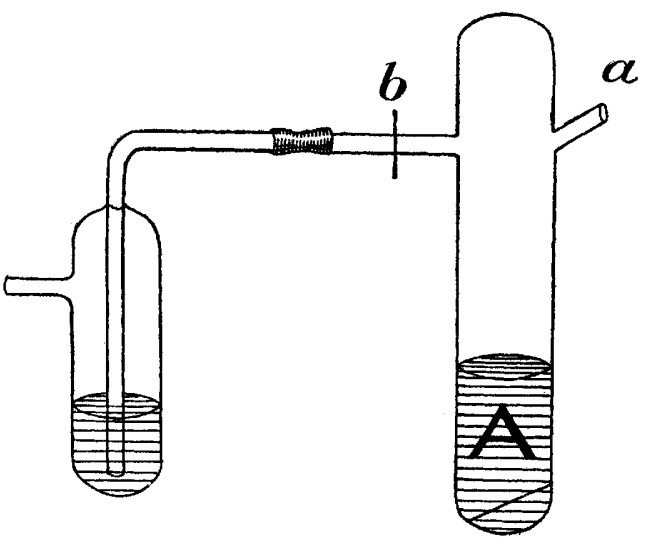

It was found by these experiments that solutions of the following substances totally inhibited oxidation, the iron being quite bright and unchanged even after several years; sodium carbonate, potassium carbonate, ammonium carbonate, ammonia, calcium hydroxide, borax, disodium hydrogen phosphate, sodium nitrite, potassium ferrocyanide, chromic acid (carefully freed from sulphuric acid), potassium chromate, and potassium dichromate. As was to be expected the formation of rust is not entirely prevented if solutions are very dilute.

If the iron is covered with platinum black, manganese dioxide, lead hydroxide, or purified animal charcoal, oxidation takes place only slowly or is stopped altogether. In the presence of calcium carbonate, however, rusting readily occurs. The amount of rusting which occurs when insoluble solids are used appears to depend partly on the extent 
to which the surface of the metal is mechanically protected from the continuous action of the liquid by the layer of powder which covers it, and partly also on the nature of the protecting solid.

Rusting is inhibited by strong solutions of sodium hydrogen carbonate (5-10 per cent.), but in dilute solutions ( 1 per cent. or less) the iron becomes covered with a brown film.

Solutions of the following salts did not prevent the iron from undergoing corrosion : sodium chloride, potassium chlorate, ferrous sulphate, potassium nitrate, potassium ferricyanide, and sodium sulphate. In the case of potassium nitrate, the action seemed to be somewhat retarded, the amount of oxide formed being less than that produced when iron is treated with water and oxygen alone. The explanation of this retarding action was afforded by the subsequent observation that potassium nitrite, a salt which inhibits rusting, had been produced during the contact of iron and potassium nitrate.

A consideration of the results of these experiments suggested that the chemical process of rusting might involve the formation of hydrogen peroxide. In support of this suggestion is the fact that rusting does not take place unless water in the liquid state is present, a condition which is favourable, if not essential, to the formation of hydrogen peroxide, whilst the supposition receives further support from the observation that those substances, such as alkalis, alkaline salts, and potassium dichromate, which prevent rusting from taking place are also known to be capable of decomposing a solution of hydrogen peroxide and therefore of interfering with its existence. On the other hand, those salts which are incapable of decomposing hydrogen peroxide have been found not to stop the process of rusting.

The remainder of this paper describes the investigation of this view of the rusting process and the experiments made with a view to elucidating the part played by hydrogen peroxide.

\section{Formation of Hydrogen Peroxide during the Oxidation of Metals.}

It is well known that hydrogen peroxide is formed during many processes in which aërial oxidation occurs, and that its presence in small quantity may usually be detected during the aërial oxidation of certain metals, for example, zinc. The production of hydrogen peroxide in these cases suggests that this substance is also formed in the rusting of iron, although direct evidence of its presence cannot be obtained with certainty. All experiments made in this direction have yielded virtually negative results. On account of its extreme delicacy for the detection of hydrogen peroxide, the titanic acid test was employed. Iron freshly reduced in hydrogen was shaken with oxygen 
and water, and the liquid, examined from time to time, showed no trace of hydrogen peroxide, but when zinc was treated under the same conditions, a distinct reaction for hydrogen peroxide was obtained in two hours. When a mixture of iron and calcium hydroxide was shaken with air and water, no trace of hydrogen peroxide or calcium peroxide could be detected. Experiments in which iron is immersed in dilute hydrogen peroxide showed that reaction is almost immediate, the peroxide being rapidly decomposed with formation of rust.

The fact that hydrogen peroxide is so readily attacked by iron, as well as by ferrous hydroxide, would certainly render the detection of peroxide difficult, assuming this substance to be an intermediate product of the rusting of iron.

Traube's experiments in connection with the production of hydrogen peroxide during the combustion of a jet of hydrogen or carbon monoxide were repeated and confirmed, a distinct reaction with titanic acid and dichromate being obtained in each case from the water on which the flame had played. When, however, a burning jet of ether vapour, a luminous gas flame, or a non-luminous gas flame was allowed to play on the surface of water either at the ordinary temperature or at zero, no trace of hydrogen peroxide could be detected. In these cases, the presence of products of incomplete combustion capable of reacting with hydrogen peroxide would prevent its detection in the free state.

The action of oxygen and water on several metals in the presence of a trace of sulphuric acid, which would promote the liberation of hydrogen, was now examined in the following manner. The metal was placed under distilled water containing a trace of sulphuric acid, and oxygen was bubbled through the liguid for some time. The mixture was then shaken and tested for hydrogen peroxide from time to time. It was found in all these instances except in that of iron. A parallel series of experiments was carried out omitting the sulphuric acid, and, except in the case of zinc, no hydrogen peroxide could be detected.

The results of the experiments made in the presence of acids were as follows :

Metal. Result.

Copper. After 48 hours, the liquid was of a faint blue colour, and gave a distinct reaction for hydrogen peroxide.

Mercury. After 2 hours, a distinct reaction for hydrogen peroxide.

Silver. After 24 hours, a trace of hydrogen peroxide was found. 
Metal.

Result.

Lead.

The liquid became milky at once, and gave a wellmarked reaction for hydrogen peroxide.

Bismuth. A well-marked reaction for hydrogen peroxide.

Tin.

The reaction was not so well marked as in the case of bismuth.

Zine.

A well-marked reaction for hydrogen peroxide.

Iron.

Although examined from time to time, no hydrogen peroxide could be detected.

Thus, hydrogen peroxide was found in every case except in that of iron. If hydrogen peroxide were produced in presence of iron, it would be at once decomposed.

In order to ascertain whether hydrogen peroxide would attack iron in the presence of a substance which inhibits ordinary rusting, metallic iron was introduced into solutions of borax and lime respectively, and hydrogen peroxide was then added. The iron in these cases remained unattacked, although the peroxide was decomposed and oxygen was evolved. If a plate of bright and highly polished steel is immersed in a strongly alkaline solution of hydrogen peroxide, decomposition of the hydrogen peroxide is extremely rapid and bubbles of oxygen are liberated on the surface of the steel, yet no rusting occurs if the alkaline solution is fairly strong.

\section{Aërial Oxidation or Rusting of Metals in General.}

By the direct action of hydrogen peroxide on metallic iron, a red, basic ferric hydroxide is produced. In order to further elucidate the general theory of rusting, other metals were examined in respect of their capacity to undergo oxidation in the air and to decompose hydrogen peroxide. It was found that in general those metals rust in air which are oxidised by hydrogen peroxide, whilst those metals which are not oxidised by hydrogen peroxide do not rust in air. The following metals belong to the former class: iron, zinc, cadmium, lead, aluminium, and bismuth. The second class contains the metals copper, platinum, silver, gold, nickel, and antimony, which neither rust appreciably in air nor react with hydrogen peroxide to form the metallic hydroxide. The rusting of the metals of the first class is found without exception to be stopped by contact with substances which are capable of decomposing hydrogen peroxide. Thus if zinc foil is placed under water in presence of oxygen, corrosion is prevented by the presence of sodium carbonate, calcium hydroxide, borax, ammonia, and potassium dichromate, whilst sodium chloride does not inhibit the action.

The results of experiments with lead and aluminium confirmed 
these conclusions, and although this series of experiments was not so complete as that carried out in the case of iron, the evidence appears sufficient to warrant the general statement that aërial oxidation or the interaction of metals with free oxygen in the presence of liquid water is prevented by solution in the water of substances which are able to decompose hydrogen peroxide.

\section{The General Theory of Rusting.}

Of the various suggestions which have been offered to explain the formation of hydrogen peroxide in the aërial oxidation or so-called "autoxidation," the two which appear the most probable are those of Hoppe-Seyler and Moritz Traube. These hypotheses are expressed by the following diagrams:

$$
\begin{aligned}
& \mathrm{Fe}+\mathrm{O}: \mathrm{O}+\mathrm{H}_{2} \mathrm{O}=\mathrm{FeO}+\mathrm{H}_{2} \mathrm{O}_{2} \text { (Hoppe-Seyler). } \\
& \mathrm{Fe}+\left(\mathrm{HO} \cdot \mathrm{H}_{2}+\mathrm{O}_{2}=\mathrm{Fe}(\mathrm{OH})_{2}+\mathrm{H}_{2} \mathrm{O}_{2}\right. \text { (Traube). }
\end{aligned}
$$

If the former theory affords the true explanation, the active oxygen attacking the iron to form the oxide, then it follows that ozone ought to attack iron even in saline solutions which decompose hydrogen peroxide, since in this reaction no hydrogen peroxide would be formed, and hence the action would not be stopped by the presence of substances which decompose it or inhibit its formation.

The reaction would be thus represented :

$$
\mathrm{Fe}+\mathrm{O} \cdot \mathrm{O}_{2}+\mathrm{H}_{2} \mathrm{O}=\mathrm{FeO}+\mathrm{O}_{2}+\mathrm{H}_{2} \mathrm{O} \text {. }
$$

On the other hand, if Traube's hypothesis is correct, ozone would not act exceptionally, since the hydrogen peroxide is assumed to be formed by "reduction" of molecular oxygen only and not by the oxidation of water. The following experiments were carried out.

Ozonised oxygen was prepared by passing purified oxygen through a Siemens' ozoniser. The tubes were filled as described previously, the oxygen being replaced by ozonised oxygen. Tubes were prepared containing the pure iron and (i) dry ozonised oxygen, (ii) ozonised oxygen and aqueous vapour, (iii) ozonised oxygen and water, (iv) ozonised oxygen and aqueous solution of sodium carbonate, ( $v$ ) ozonised oxygen and solution of potassium dichromate. In each case the ozonised oxygen behaved precisely as ordinary oxygen does under the same conditions. A series of parallel experiments in which iron was absent showed that ozone is only slowly decomposed under these conditions, ozone in the presence of water and sodium carbonate not being totally decomposed until after three days. These experiments prove, therefore, that ozonised oxygen behaves towards iron as ordinary 
oxygen does and that the active oxygen of ozone cannot initiate the rusting of iron.

Action of Nitrous Oxide.-Again, if the theory which supposes direct action of iron on the oxygen is correct, it is probable that the oxygen could be replaced by nitrous oxide which, as is well known is readily separated into nitrogen and oxygen. The reaction might proceed thus :

$$
\mathrm{Fe}+\mathrm{O} \mathrm{N}_{2}+\mathrm{H}_{2} \mathrm{O}=\mathrm{FeO}+\mathrm{N}_{2}+\mathrm{H}_{2} \mathrm{O},
$$

and rusting should therefore take place in the absence of free oxygen. Two experiments in which pure iron was left in contact with water and nitrous oxide in the absence of free oxygen were carefully carried out in the manner already described, but no rusting occurred in either case.

On the other hand, if it is supposed with Traube that the metal first attacks the water liberating hydrogen, it ought to be possible to replace the oxygen by some reducible substance capable of reacting with the liberated hydrogen. Rusting should then proceed in the absence of free oxygen.

Experiments were made in which potassium ferricyanide, nitroethane, nitrobenzene, methyl alcohol, free hydroxylamine, and potassium nitrate, respectively, were included in the tube containing pure iron and pure water, the remainder of the tube being full of hydrogen. In order to prove the complete absence of oxygen, the hydrogen, before being allowed to enter, was passed through a mixture of pure iron and water only, contained in a tube which was afterwards sealed up; since no rusting took place in this tube, the absence of oxygen was verified. The results of the experiments were as follows. In the case of potassium ferricyanide, the liquid assumed a yellowish-green colour, whilst the surface of the iron became coated with a blue substance; after a time the action ceased and no further change was observed. With nitroethane, ordinary rust was produced on the iron and the liquid became dark in colour. In the case of hydroxylamine, oxidation of the iron occurred and bubbles of gas were evolved. In another experiment in which the iron was exposed to the action of hydroxylamine and water in a vacuum, the same action was noticed. With potassium nitrate and nitrobenzene, the iron remained quite bright.

The results of these experiments show therefore that the free oxygen can be replaced by potassium ferricyanide, nitroethane, or hydroxylamine, and that under these conditions rusting of iron takes place in the absence of free oxygen.

Experiments were also made in which pure iron and oxygen were left in contact with dry ether instear of water. In this case no 
rusting occurred. It is therefore concluded from the results of all these experiments that liquid water is essential for the rusting of iron, and that the chemical action involved is the reduction of the water by the iron, the hydrogen thus formed going to produce hydrogen peroxide, which, reacting with ferrous oxide first formed, produces the form of ferric hydroxide known as iron rust.

$$
\begin{gathered}
\mathrm{Fe}+\mathrm{OH}_{2}=\mathrm{FeO}+\mathrm{H}_{2} ; \mathrm{H}_{2}+\mathrm{O}_{2}=\mathrm{H}_{2} \mathrm{O}_{2} ; \\
2 \mathrm{FeO}+\mathrm{H}_{2} \mathrm{O}_{2}=\mathrm{Fe}_{2} \mathrm{O}_{2}(\mathrm{OH})_{2} .
\end{gathered}
$$

\section{The Composition of Iron Rust.}

The composition of iron rust is somewhat variable and depends on the purity of the iron from which it is derived and on the conditions under which the rusting has occurred. It contains a small and variable proportion of ferrous iron, and is usually magnetic. The analysis of a number of specimens of rust from different sources produced under different conditions and dried by exposure to the air gave the following results :

\section{Rust obtained from Commercial Iron.}

\begin{tabular}{|c|c|c|c|}
\hline (Ferrous.. & $\begin{array}{c}\text { Specimen } 1 . \\
60.93\end{array}$ & Specimen 2. & Mean. \\
\hline 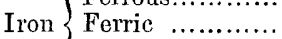 & 0.13 & - & - \\
\hline Total & $61 \cdot 06$ & $60 \cdot 78$ & $60 \cdot 92$ \\
\hline Hydrogen & $1 \cdot 01$ & $1 \cdot 03$ & $1 \cdot 02$ \\
\hline Oxygen... & $34 \cdot 35$ & $34 \cdot 55$ & $34 \cdot 45$ \\
\hline Carbon dioxide ......... & $0 \cdot 15$ & 0.18 & 0.17 \\
\hline (.............. & 0.006 & - & 一 \\
\hline Insoluble matter .. & $3 \cdot 20$ & $3 \cdot 46$ & $3 \cdot 33$ \\
\hline
\end{tabular}

A. Rust collected from iron apparatus rusted in the laboratory.

B. Rust from commercial iron exposed to the action of air and water.

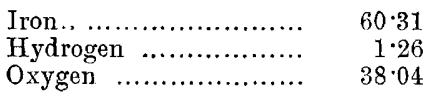

\section{Calculated for $\mathrm{Fe}_{2} \mathrm{O}_{2}(\mathrm{OH})_{3}$.}

$1 \cdot 3$

$35 \cdot 9$

C. Rust from commercial iron exposed to the action of moist air.

An opportunity presented itself of examining a specimen of rust formed from iron during exposure to air for many years. The specimen was obtained from an iron railing which had been for thirty years in an exposed position on the sea coast within twenty yards of the sea. On analysis, a piece of the entire railing furnished the following results : 


\begin{tabular}{|c|c|c|}
\hline & & Calculated for $\mathrm{F}$ \\
\hline (............. & $59 \cdot 25$ & - \\
\hline Iron $\{$ Ferrous...$\ldots \ldots \ldots$ & $3 \cdot 25$ & - \\
\hline Total $\ldots \ldots \ldots \ldots$ & $62 \cdot 50$ & $62 \cdot 8$ \\
\hline Hydrogen ................ & $1 \cdot 17$ & $1 \cdot 3$ \\
\hline Oxygen & $35 \cdot 70$ & $35 \cdot 9$ \\
\hline Insoluble matter .......... & $0 \cdot 60$ & - \\
\hline
\end{tabular}

It thus appears that this natural rust contains a little ferrous oxide, and, allowing for this, its composition would correspond closely with the formula $\mathrm{Fe}_{2} \mathrm{O}_{2}(\mathrm{OH})_{2}$, which, on the whole, expresses the usual composition of iron rust. It is an interesting coincidence, if nothing more, that a substance of this formula would be formed by the union of two molecules of ferrous oxide with one of hydrogen peroxide.

\section{Rust obtained in Experiments with Highly Purified Iron.}

D. Rust from pure iron exposed to the action of air and water.

E. Rust from pure iron exposed to the action of pure air and 5 per cent. sodium chloride solution.

F. Rust from pure iron in contact with pure oxygen and pure water (results previously quoted on page 1556).

\begin{tabular}{|c|c|c|c|c|c|c|c|}
\hline & D. & D. & E. & F. & F. & Average. & $\begin{array}{l}\text { Calculated for } \\
\mathrm{Fe}_{2} \mathrm{O}_{2}(\mathrm{OH})_{2} \text {. }\end{array}$ \\
\hline Iron & $61 \cdot 39$ & $61 \cdot 25$ & $61 \cdot 2$ & $62 \cdot 16$ & 622 & $61 \cdot 64$ & $62 \cdot 8$ \\
\hline Hydrogen.. & $1 \cdot 3$ & $1 \cdot 26$ & $1 \cdot 2$ & $1 \cdot 4$ & $1 \cdot 2$ & $1 \cdot 22$ & $1 \cdot 3$ \\
\hline Oxygen .... & $37 \cdot 31$ & $37 \cdot 49$ & $37 \cdot 6$ & $35 \cdot 97$ & $36 \cdot 6$ & $36 \cdot 99$ & $35 \cdot 9$ \\
\hline
\end{tabular}

It will be noticed that the entire series of results are remarkably concordant for a substance produced under varying conditions. Exact agreement, as is to be expected, is only found in the case of rust formed under the same conditions. The results obtained from rust formed by pure iron in pure oxygen and water agree best with the formula $\mathrm{Fe}_{2} \mathrm{O}_{2}(\mathrm{OH})_{2}$, whilst those obtained from rust formed by the corrosion of impure iron in air are not far removed from the same composition.

\section{The Reaction between Iron and Water at High Temperatures.}

It is well known that at temperatures above $100^{\circ}$ reaction occurs between iron and water with evolution of hydrogen and formation of an iron oxide It seemed to be of interest to ascertain whether the action at higher temperatures than that of the air would be affected by alkalis. The following experiments were made. 
Pieces of iron were placed in two tubes, one of which contained water, whilst the other contained an aqueous solution of sodium carbonate. The tubes were exhausted, and were afterwards sealed and heated at $150-200^{\circ}$ for some hours. The iron was much corroded, and on opening the tubes a pressure of the same magnitude was found in each case, a large amount of gas having been produced.

In another experiment, a tube containing iron, oxygen, and solution of sodium carbonate was heated at $100^{\circ}$ for three hours. The metal was corroded, and the presence of hydrogen was proved.

These results show that the corrosion of iron under these conditions proceeds with evolution of hydrogen and is not affected by the presence of sodium carbonate. The process is therefore quite distinct from that of ordinary " rusting."

\section{The Influence of Carbon Dioxide on Rusting.}

It has been shown already that the statement of Crace Calvert, Crum Brown, and others, that iron will not rust in the absence of carbon dioxide is erroneous. Since, however, there is no doubt that carbon dioxide does take part in the ordinary atmospheric corrosion of iron, it was considered desirable to ascertain experimentally its precise influence.

It has been stated by Crum Brown (loc. cit.) that carbonic acid reacts with metallic iron with evolution of hydrogen and formation of ferrous carbonate or bicarbonate, but the recorded experimental evidence on the subject is unsatisfactory, and further experiments were therefore made. Pure dry hydrogen was passed over ferric oxide heated in a glass tube, and the reduced iron was allowed to cool in an atmosphere of hydrogen. The hydrogen was then replaced by carbon dioxide, and the reduced iron was quickly transferred to thin glass tubes, which were afterwards sealed. One of the tubes containing reduced iron was introduced into a solution of carbon dioxide under pressure contained in a well-stoppered bottle. The tube was broken by shaking the bottle, which was then set aside for several weeks. At the end of this time the gas was withdrawn from the bottle, and on examination was found to consist largely of hydrogen. The liquid contained iron dissolved as carbonate. In another experiment, the reduced iron was left in contact with the solution of carbon dioxide for a year. In this case, the remaining gas was found to be hydrogen nearly free from carbon dioxide, which had gone to form dissolved ferrous carbonate.

In the following experiments, pieces of pure sheet iron were placed in tubes, and were left for several months in contact with water and mixtures of carbon dioxide and oxygen. The method adopted for fill- 
ing the tubes was that which has been previously described (page 1553). The results show that the carbonic acid, when present in considerable quantity, first reacts with the iron in the presence of water to form a soluble bicarbonate, which is then oxidised by the oxygen present, and is precipitated as a basic hydroxide containing a varying amount of carbonate. The results obtained are described in the following table :

Gas used in tube.

(1) Oxygen with a small proportion of carbon dioxide.

(2) Oxygen : carbon dioxide. $4: 1$,

(3) Oxygen : carbon dioxide. $3: 2$.

(4) Oxygen and carbon dioxide in equal volumes.

(5) Oxygen : carbon dioxide.

\section{$2: 3$.}

(6) Pure carbon dioxide.
Results.

The iron rapidly became coated with a film, and the liquid became turbid, the rust formed having the appearance of ordinary rust.

No change was visible at first, the iron remaining quite bright, but after four days a red, flaky substance was deposited on the side of the tube and on the edge of the metal nearest the surface of the liquid. This deposit gradually increased, until after two years there was a large amount of light brown sediment present and a brown film on the sides of the tube. The iron itself was pitted and of a steel-grey colour.

A red deposit was formed on the side of the tube, and in four months a considerable quantity of rust was produced.

The iron remained bright at first, but later dark brown scales were deposited on the sides of the tube and on the surface of the liquid, the iron becoming pitted and of a dark colour.

Appearance very much like that of (4).

The surface of the iron was pitted, and a white, crystalline deposit formed on the sides of the tube.

The rust produced in experiment (2), after drying by exposure to the air, furnished the following results on analysis:

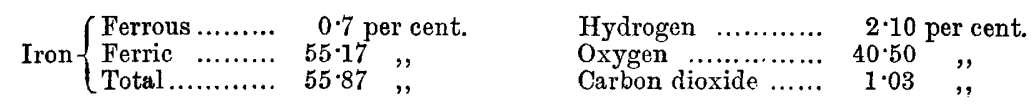

It is evident that the product of this action is very different in 
composition from that formed in the process of rusting in oxygen or in ordinary air.

These results lead to the conclusion that carbonic acid plays a comparatively unimportant part in ordinary atmospheric rusting of iron, and that the chemical change is chiefly concerned with the interaction of iron, water, and oxygen. If this interaction cannot occur through the presence of certain salts, carbonic acid produces little or no rust even when oxygen is present in considerable quantity.

\section{Crace Calvert's Experiments.}

In the course of the investigation on the influence of carbon dioxide on rusting, it was thought desirable to repeat certain experiments described by Crace Calvert (loc. cit.), partly referred to earlier in this paper. In these experiments water, oxygen, carbon dioxide, and solutions of certain salts were used, and a strip of pure iron was half immersed in the liquid, whilst the other half was in contact with the gas. The following results were obtained.

\section{(1) In Water and Pure Oxygen.}

Portion of iron in water.-In a few minutes spots appeared which gradually developed into ordinary rust.

Portion of iron in oxygen.-The metal remained bright at first, then became dull and rusted at the edges.

Crace Calvert states that the iron in the water rusts, whilst that in contact with the gas does not.

(2) In Water and a Mixture of Oxygen and Carbon Dioxide.

Iron in water.-In a few minutes a green substance was formed which soon became red, and the sides of the tube became coated with a red substance.

Iron in gas.-The metal remained bright at first, but afterwards became rusted in places.

Crace Calvert asserts that the entire surface of the iron inside and outside the water becomes coated with rust.

(3) In Air and a 5 per cent. Solution of Sodium Carbonate.

Iron in liquid.-The surface remained quite bright.

Iron in the gas. - A few spots of rust appeared on the surface.

Crace Calvert asserts that no rusting occurs on any part of the iron in this experiment.

It will be seen that these results are not in agreement with those 
obtained by Crace Calvert. They coincide, however, with those furnished by the experiments described in the earlier part of this paper. The action produced is evidently dependent on the combined action of oxygen and liquid water. Carbonic acid contributes to the effect, since in the presence of carbonic acid and oxygen the iron is attacked, an oxycarbonate of iron being initially formed. The evidence gained from the analysis of different specimens of ordinary rust shows, however, that any oxycarbonate of iron formed in this way during rusting subsequently undergoes gradual decomposition, with the result that a hydrated ferric oxide, containing only a trace of carbonate, ultimately remains. In ordinary circumstances, the proportion of rust due to this reaction must be necessarily very small owing to the minute proportion of carbon dioxide in the atmosphere.

The following experiments were made in order to gain some idea of the extent to which carbon dioxide accelerates the process of rusting.

Pieces of clean purified iron were submitted to the action of water, carbon dioxide, and air, the same conditions being observed in each case.

A saturated solution of carbon dioxide was prepared by passing a current of the gas, generated by the action of hydrochloric acid on marble, through freshly-boiled, distilled water. The carbon dioxide was purified by leading it through a wash-bottle containing sodium hydrogen carbonate. The carbonic acid solution was proved to be free from hydrochloric acid by testing it with silver nitrate. It contained about its own volume of carbon dioxide in solution.

Four flasks $(A, B, C$, and $D)$ of one litre capacity were taken and provided with well-fitting corks.

$A$. One hundred c.c. of the carbon dioxide solution were put into the flask and a current of the gas was passed in. While the flask was still full of carbon dioxide, three pieces of clean sheet iron (each about $3 \mathrm{~cm} . \times 2 \mathrm{~cm}$. in size) were dropped in, and the cork was quickly inserted.

$B$. In this flask were placed 50 c.c. of the carbon dioxide solution, 50 c.c. of well-boiled, distilled water, and three pieces of iron of the same size as those used in the preceding experiment, the remainder of the flask being filled with air.

C. In this flask were placed 10 c.c. of the carbon dioxide solution, 90 c.c. of air-free water, three pieces of iron of the same size as before, the remainder of the flask being filled with air.

$D$. In this flask were placed 100 c.c. of air-free water and three pieces of iron, the remaining space being filled with air. 


\section{Results.}

In the course of 10 or 15 minutes after the experiments had been started, the surface of the metal in flask $A$ was thickly coated with bubbles. In flask $B$ the surface was covered with bubbles of a smaller size, whilst in $C$ the bubbles were extremely small. In flask $D$ no bubbles were visible.

After 19 hours, the solution in flask $A$ was clear and colourless, and the surface of the metal was thickly coated with bubbles. In $B$, the solution was faintly yellow and opalescent ; a trace of rust appeared on the surface of the iron, which was coated with bubbles. The liquid in $C$ was pale yellow in colour, and a small amount of reddish-brown rust was suspended in it; a little rust (more than in the case of $B$ ) and numerous small bubbles appeared on the surface of the metal. In flask $D$, the metal was thickly coated with rust of the usual appearance.

Although it has been found that quantitative experiments on these lines cannot be made with absolutely accuracy, it was thought worth while to determine the amount of iron attacked in each case, the rust being carefully brushed from the surface of the metal and washed into the liquid in the flask. Sulphuric acid was added to the liquid, and the reduction of the iron to the ferrous state was effected by means of zinc. The solution was afterwards titrated with $N / 10$ potassium permanganate. The following results were obtained:

$\begin{array}{ccccc}\text { Flask. } & \text { A. } & \text { B. } & \text { C. } & \text { D. } \\ \text { Amount of iron attacked, in grams } & 0.005 & 0.004 & 0.0022 & 0.0012\end{array}$

These results show that a solution of carbonic acid in the absence of oxygen $(A)$ is able to dissolve metallic iron, even when the metal is not in a finely-divided condition, the iron remaining in solution as carbonate ; the action is, however, very slow, and even in the presence of water saturated and surrounded with an atmosphere of carbon dioxide the amount of iron dissolved is very small. In the presence of air and about half the quantity of carbonic acid $(B)$, the action is about the same (actually rather smaller in this experiment), but some of the iron attacked appears as rust. In the presence of more air and less carbonic acid $(C)$, the action is considerably reduced, although the amount of rust formed is greater than when the air contains only the usual proportion of carbon dioxide $(D)$.

Since it has been shown that rusting proceeds rapidly in the entire absence of carbon dioxide, it is evident from these experiments that the minute quantity of carbon dioxide in the air can play only a small and subordinate part in the ordinary rusting of iron. 
Three notes on the corrosion of iron and zinc have been recently published by Moody (Proc., 1903, 19, 157, 239, 273), in which he reverts to the old theory that the "rusting" of these metals is due entirely to the action of carbonic acid. This hypothesis has been proved by the results of the present investigation to be quite untenable, since it has been shown that rusting can take place in the absence of carbonic acid.

The retarding influence of certain substances on the production of rust is considered by Moody to be wholly due to the influence which they exert on the absorption of carbon dioxide. It is stated that although iron when immersed in 1 per cent. solution of chromium trioxide remains bright for some weeks, yet, nevertheless, the metal is slowly being dissolved and that eventually its surface becomes coated with rust. The result is quite contrary to the experience of the present authors, who have in their possession a specimen of iron which has been in contact with a solution of pure chromic acid for nearly ten years and still remains quite bright. Aqueous potassium dichromate similarly inhibits the rusting of iron.

It has been pointed out by Moody that distilled hydrogen peroxide is without action on iron. It appears, however, that this is merely an example of a reaction which is hindered by the employment of very pure materials, for if a small quantity of sodium chloride is added to the solution, the iron is rapidly attacked and a red, flocculent rust is produced, as is the case when ordinary aqueous hydrogen peroxide is used. The account given by Giorgis (Gazzetta, 1891, 21, 510) of the action of hydrogen peroxide on magnesium in the absence of carbon dioxide appears to have been misread by Moody. The results described by Giorgis indicate that hydrogen peroxide attacks magnesium in the absence of carbon dioxide.

\section{Formation of Rust by Electrolytic Action.}

When iron is impure, as is always the case with commercial specimens, or when another metal is present, electrolytic action is liable to take place. It is also known that iron and iron-rust can act as a voltaic couple. It therefore seemed desirable to ascertain the influence of this electrolytic action on the ordinary process of rusting, and a series of experiments was made to determine the nature of the action and the effect of saline solutions on it.

In the first place it was proved that solutions of sodium carbonate and potassium dichromate do not affect the electrolytic decomposition of water by a zinc-copper couple. The experiments were conducted as follows. Three tubes were taken containing water, a solution of sodium carbonate, and a solution of potassium dichromate respectively. 
To each tube a manometer was attached (Figure 4). Zinc-copper couple (the same weight in each case) was added to the liquid in the tube. In each instance, an internal pressure was set up due to the evolution oî a gas, probably hydrogen, and the reaction proceeded in the same way.

Two general cases were now studied, in one of which iron acted as the electro-positive, and in the other as the electro-negative, element. The couples were prepared by wrapping a strip of the second metal round the iron (Figure 5). Experiments were carried out with several couples but the best results were obtained with the zinc-iron couple and the iron-platinum couple. Confirmatory results were obtained with other couples.

The zinc-iron couples were immersed in solutions of sodium carbonate, sodium hydrogen carbonate, borax, potassium chromate, potassium di-

FIG. 4.

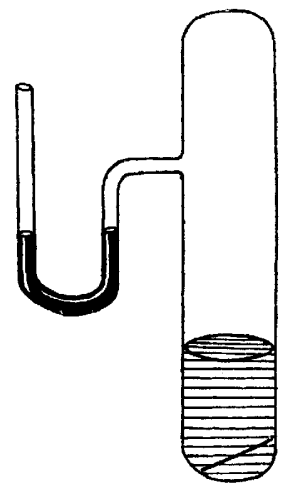

FIG. 5 .

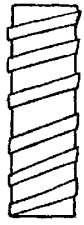

chromate, chromic acid, and potassium ferrocyanide. In each case the zinc (the electro-positive element) was oxidised, especially on the under surface, where it was in contact with the iron, whilst the iron remained bright.

Since it has been proved that the solutions employed inhibit the ordinary corrosion of zinc, it follows that the reaction is one of electrolytic decomposition of water involving oxidation of the positive element and evolution of hydrogen.

The iron-platinum couples were placed in solutions of the seven substances just enumerated. In every instance, the iron became rusted only where it was in contact with the platinum, the other part remaining unoxidised. Here again the results are in conformity with the view that the observed change is due to an electrolytic process, the positive element (iron) being attacked by the cation (oxygen), whilst 


\section{THE RUSTING OF IRON.}

the hydrogen is evolved from the negative element (platinum), the presence of the salts preventing the ordinary corrosion of the iron by dissolved oxygen.

It is well known that the rusting of iron is partially prevented by contact with zinc, and, in view of the results of the experiments just described, it seems probable that any iron rust formed by atmospheric corrosion is reduced by the hydrogen evolved in the electrolytic decomposition of water by the zinc-iron couple. This conclusion is confirmed by the following experiments :

1. A zinc-iron" couple was placed in a tube with a pressure-gauge attached as already described and in contact with air and pure water. No gas was evolved, the zinc became oxidised, but the iron remained quite bright.

2. A piece of iron, rusty on one side and bright on the other, was

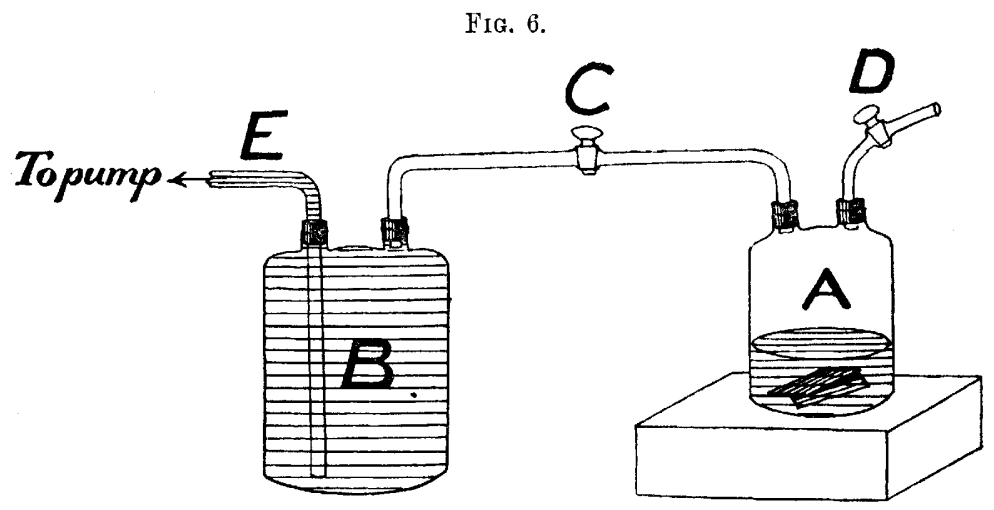

wrapped round with zinc foil and placed in a tube of water. The tube was rendered vacuous and then sealed. A few bubbles of gas were evolved, the zinc became much oxidised, and the rust on the iron slowly disappeared.

3. Water was submitted to electrolysis, platinum being used as the cathode and a zinc-iron couple as the anode. Hydrogen was evolved at the platinum electrode, whilst zinc oxide with only a trace of iron oxide was produced at the anode.

These experiments show that the protection of iron by zinc is due to the liberation of hydrogen which tends to reduce any oxide of iron formed by ordinary atmospheric oxidation.

It now became of interest to ascertain whether this electrolytic process proceeds in the ordinary "rusting" of pure iron and, if so, to determine its extent by estimating the amount of hydrogen evolved. The experiment was conducted in the following manner. A quantity

VOL. LXXXVII. 
of pure iron, weighing 26 grams, and 260 c.c. of water saturated with oxygen were introduced into a small Woulff's bottle, $A$ (Figure 6). This vessel was connected with a larger bottle, $B$, which was filled with water and attached to an air-pump by the tube $E$. The gas in bottle $A$ was drawn off from time to time into bottle $B$, and a further supply of air was admitted through tap $D$. When 0.3 gram of rust had been formed, the whole of the gas was transferred to $B$ and examined for the presence of hydrogen by leading it through a capillary tube containing heated platinised asbestos. The volume of the gas was measured both before and after this operation. No diminution in volume occurred and hence no hydrogen was present.

It is therefore proved that in the ordinary atmospheric rusting of pure iron electrolytic action does not occur, and that no hydrogen is set free in the process.

We consider that the results of this investigation prove conclusively that iron, oxygen, and liquid water are alone necessary for the rusting of iron to take place. Under atmospheric conditions, carbon dioxide plays a quite subordinate part in the process. One of the simplest representations of the chemical action between iron, oxygen, and liquid water presupposes the intermediate formation of hydrogen peroxide. Although the actual production of this substance has not been demonstrated, a considerable body of experimental evidence has been obtained in favour of its temporary formation. We have also shown that the conditions of the spontaneous oxidation of iron known as rusting present peculiarities which are not shared by other processes in which iron undergoes oxidation with formation of a ferric hydroxide.

Scientific Departanen, IMPERIAL INSTITUTE. 